Supporting Information for:

\title{
Investigation into an Unexpected Impurity: A Practical Approach to Process Development for the Addition of Grignard Reagents to Aldehydes
}

\section{Using Continuous Flow Synthesis}

Masahiro Hosoya,* Shogo Nishijima, Noriyuki Kurose

API R\&D Laboratory, CMC R\&D Division, Shionogi and Co., Ltd., 1-3, Kuise Terajima 2-chome, Amagasaki, Hyogo 660-0813, Japan

Contents:

S2: HPLC methods for preparation of $\mathbf{2 a - 2 h}$, and $\mathbf{3 a}$

S3-4: Procedures of batch synthesis to synthesize 2a

S5: ${ }^{1} \mathrm{H}$ NMR and ${ }^{13} \mathrm{C}$ NMR spectra of 2a

S6-10: ${ }^{1} \mathrm{H}$ NMR, ${ }^{13} \mathrm{C}$ NMR, and ${ }^{1} \mathrm{H}-{ }^{1} \mathrm{H}$ COSY, ${ }^{1} \mathrm{H}^{-13} \mathrm{C}$ HMBC, ${ }^{1} \mathrm{H}_{-}{ }^{13} \mathrm{C}$ HSQC spectra of 3a

S11-12: Input amount and isolated yield of $\mathbf{2} \mathbf{b}-\mathbf{2} \mathbf{h}$ with continuous flow synthesis

S13-19: ${ }^{1} \mathrm{H}$ NMR and ${ }^{13} \mathrm{C}$ NMR spectra of $\mathbf{2 b}-\mathbf{2 h}$

S20: Heat flow about addition of MeMgBr to 1a

S21-22: Justification of calorimetry analysis combined with continuous flow system and RC1e

S23-24: Simulation details based on the heat of reaction

S25-26: Detailed description of the equipment used for continuous flow synthesis

S27: Procedure of running for a long duration 


\section{HPLC method for preparation of $2 \mathrm{a}, 2 \mathrm{c}-2 \mathrm{~h}$, and $3 \mathrm{a}$}

Column: Waters Xbridge C18, $4.6 \times 150 \mathrm{~mm}, 5 \mu \mathrm{m}$

Mobile phase A: 0.1\% HCOOH in purified water. Mobile phase B: MeCN.

B concentration (Gradient): 0.0-12.0 min 15-90\%, 12.0-15.0 min 90\%, 15.0-15.1 min 90-15\%, 15.1-20.0 $\min 15 \%$.

Flow rate: $1.0 \mathrm{~mL} / \mathrm{min}$

Injection volume: $10 \mu \mathrm{L}$

Column temperature: $35^{\circ} \mathrm{C}$

Wavelength: $254 \mathrm{~nm}$

Sample was diluted with $\mathrm{MeCN} / \mathrm{H}_{2} \mathrm{O}(80 / 20(\mathrm{v} / \mathrm{v}))$ containing $0.1 \mathrm{vol} \%$ of $\mathrm{HCOOH}$

\section{HPLC method for preparation of $\mathbf{2 b}$}

Column: Waters Xbridge C18, $4.6 \times 150 \mathrm{~mm}, 5 \mu \mathrm{m}$

Mobile phase A: $0.1 \% \mathrm{HCOOH}$ in purified water. Mobile phase B: MeCN.

B concentration (Gradient): 0.0-32.0 min 15-90\%, 32.0-35.0 min 90\%, 35.0-35.1 min 90-15\%, 35.1-40.0 $\min 15 \%$.

Flow rate: $1.0 \mathrm{~mL} / \mathrm{min}$

Injection volume: $10 \mu \mathrm{L}$

Column temperature: $35^{\circ} \mathrm{C}$

Wavelength: $254 \mathrm{~nm}$

Sample was diluted with $\mathrm{MeCN} / \mathrm{H}_{2} \mathrm{O}(80 / 20(\mathrm{v} / \mathrm{v}))$ containing $0.1 \mathrm{vol} \%$ of $\mathrm{HCOOH}$ 


\section{Procedures of batch synthesis to synthesize 2a}

\section{Entry 1 in Table 1}

MeMgBr in THF (5.7 mL, $1.07 \mathrm{mmol} / \mathrm{mL}, 1.0$ equiv) was added into a 4-neck flask containing THF (10 $\mathrm{mL})$ with a stirring bar. A solution of $1 \mathbf{a}(1.00 \mathrm{~g})$ in THF $(8 \mathrm{~mL})$ was added to the prepared solution of $\mathrm{MeMgBr}$ at $25^{\circ} \mathrm{C}$ for 10 minutes and washed with THF (2 mL). A part of the reaction mixture was poured into $\mathrm{MeCN} / \mathrm{H}_{2} \mathrm{O}(80 / 20(\mathrm{v} / \mathrm{v}))$ containing $0.1 \mathrm{vol} \%$ of $\mathrm{HCOOH}$ directly and diluted in a measuring flask to measure quantitative yield.

\section{Entry 2 in Table 1}

Two solutions were fed by the Vapourtec V-3; solution A was a solution of 1a in 10 volumes of THF (0.559 $\mathrm{mmol} / \mathrm{mL}$ ) and solution B was MeMgBr in THF (1.07 mmol/mL). Flow rate of solution A was set as 1.09 $\mathrm{mL} / \mathrm{min}$, and that of solution B was set as $0.57 \mathrm{~mL} / \mathrm{min}$ so that the equivalent of $\mathrm{MeMgBr}$ would be adjusted to 1.00 equiv. Two solutions were poured into a 4-neck flask containing THF (10 mL) with a stirring bar at $25^{\circ} \mathrm{C}$ for 11 minutes. Input amount of the starting material 1a was defined as $1.10 \mathrm{~g}(0.559$ $\mathrm{mmol} / \mathrm{mL} \times 1.09 \mathrm{~mL} / \mathrm{min} \times 11 \mathrm{~min} \times 164.16 \mathrm{mg} / \mathrm{mmol}$ ). A part of the reaction mixture was poured into $\mathrm{MeCN} / \mathrm{H}_{2} \mathrm{O}(80 / 20(\mathrm{v} / \mathrm{v}))$ containing $0.1 \mathrm{vol} \%$ of $\mathrm{HCOOH}$ directly and diluted in a measuring flask to measure quantitative yield.

\section{Entry 3 in Table 1}

MeMgBr in THF (2.8 mL, $1.07 \mathrm{mmol} / \mathrm{mL}, 1.0$ equiv) was added to a solution of $1 \mathbf{a}(0.50 \mathrm{~g})$ in THF (10 $\mathrm{mL})$ at $25^{\circ} \mathrm{C}$ for $10 \mathrm{sec}$ and washed with THF (1 mL). A part of the reaction mixture was poured into $\mathrm{MeCN} / \mathrm{H}_{2} \mathrm{O}(80 / 20(\mathrm{v} / \mathrm{v}))$ containing $0.1 \mathrm{vol} \%$ of $\mathrm{HCOOH}$ directly and diluted in a measuring flask to measure quantitative yield.

\section{Entry 4 in Table 1}

MeMgBr in THF (5.7 mL, $1.07 \mathrm{mmol} / \mathrm{mL}, 1.0$ equiv) was added to a solution of $1 \mathbf{a}(1.00 \mathrm{~g})$ in THF (15 $\mathrm{mL})$ at $25^{\circ} \mathrm{C}$ for $10 \mathrm{~min}$ and washed with THF (5 mL). A part of the reaction mixture was poured into $\mathrm{MeCN} / \mathrm{H}_{2} \mathrm{O}(80 / 20(\mathrm{v} / \mathrm{v}))$ containing $0.1 \mathrm{vol} \%$ of $\mathrm{HCOOH}$ directly and diluted in a measuring flask to measure quantitative yield.

\section{Entry 5 in Table 1}

MeMgBr in THF (5.7 mL, $1.07 \mathrm{mmol} / \mathrm{mL}, 1.0$ equiv) was added to a solution of $1 \mathbf{a}$ (1.00 g) in THF (15 $\mathrm{mL})$ at $25^{\circ} \mathrm{C}$ for $60 \mathrm{~min}$ and washed with THF (5 mL). A part of the reaction mixture was poured into $\mathrm{MeCN} / \mathrm{H}_{2} \mathrm{O}(80 / 20(\mathrm{v} / \mathrm{v}))$ containing 0.1 vol\% of $\mathrm{HCOOH}$ directly and diluted in a measuring flask to measure quantitative yield. 


\section{Entry 6 in Table 1}

MeMgBr in THF (5.7 mL, $1.07 \mathrm{mmol} / \mathrm{mL}, 1.0$ equiv) was added to a solution of $1 \mathbf{a}(1.00 \mathrm{~g})$ in THF (15 $\mathrm{mL})$ at $-10^{\circ} \mathrm{C}$ for $10 \mathrm{~min}$ and washed with THF (5 mL). A part of the reaction mixture was poured into $\mathrm{MeCN} / \mathrm{H}_{2} \mathrm{O}(80 / 20(\mathrm{v} / \mathrm{v}))$ containing 0.1 vol\% of $\mathrm{HCOOH}$ directly and diluted in a measuring flask to measure quantitative yield.

\section{Entry 7 in Table 1}

MeMgBr in THF (5.7 mL, $1.07 \mathrm{mmol} / \mathrm{mL}, 1.0$ equiv) was added to a solution of $\mathbf{1 a}(1.00 \mathrm{~g})$ in THF (15 $\mathrm{mL})$ at $-60^{\circ} \mathrm{C}$ for $10 \mathrm{~min}$ and washed with THF $(5 \mathrm{~mL})$. The reaction mixture was stirred at $-60^{\circ} \mathrm{C}$ for 30 min. The reaction mixture was heated to $5^{\circ} \mathrm{C}$ and stirred for $10 \mathrm{~min}$. A part of the reaction mixture was poured into $\mathrm{MeCN} / \mathrm{H}_{2} \mathrm{O}(80 / 20(\mathrm{v} / \mathrm{v}))$ containing $0.1 \mathrm{vol} \%$ of $\mathrm{HCOOH}$ directly and diluted in a measuring flask to measure quantitative yield. 

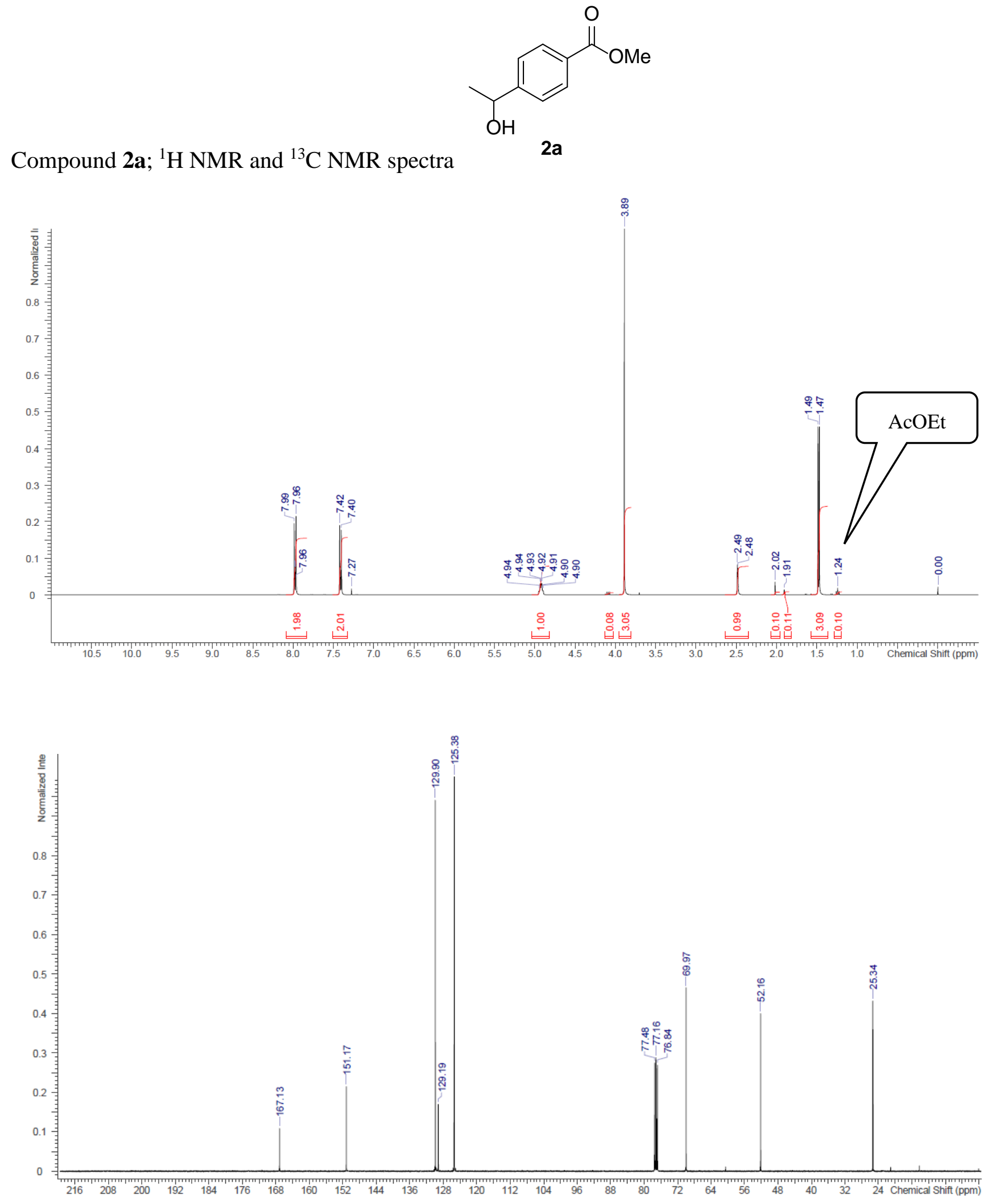


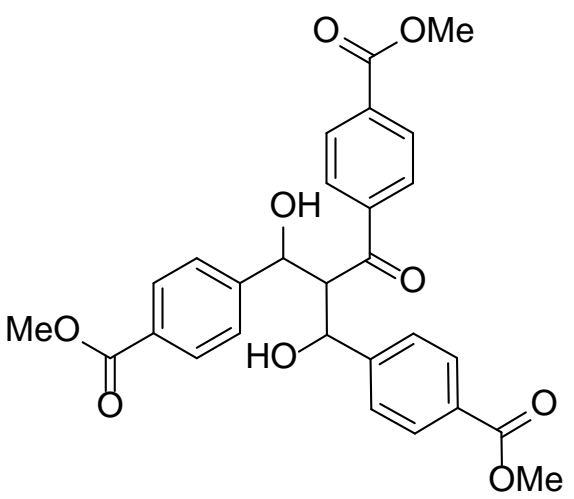

Compound 3a; ${ }^{1} \mathrm{H}$ NMR and ${ }^{13} \mathrm{C}$ NMR spectra

$3 a$

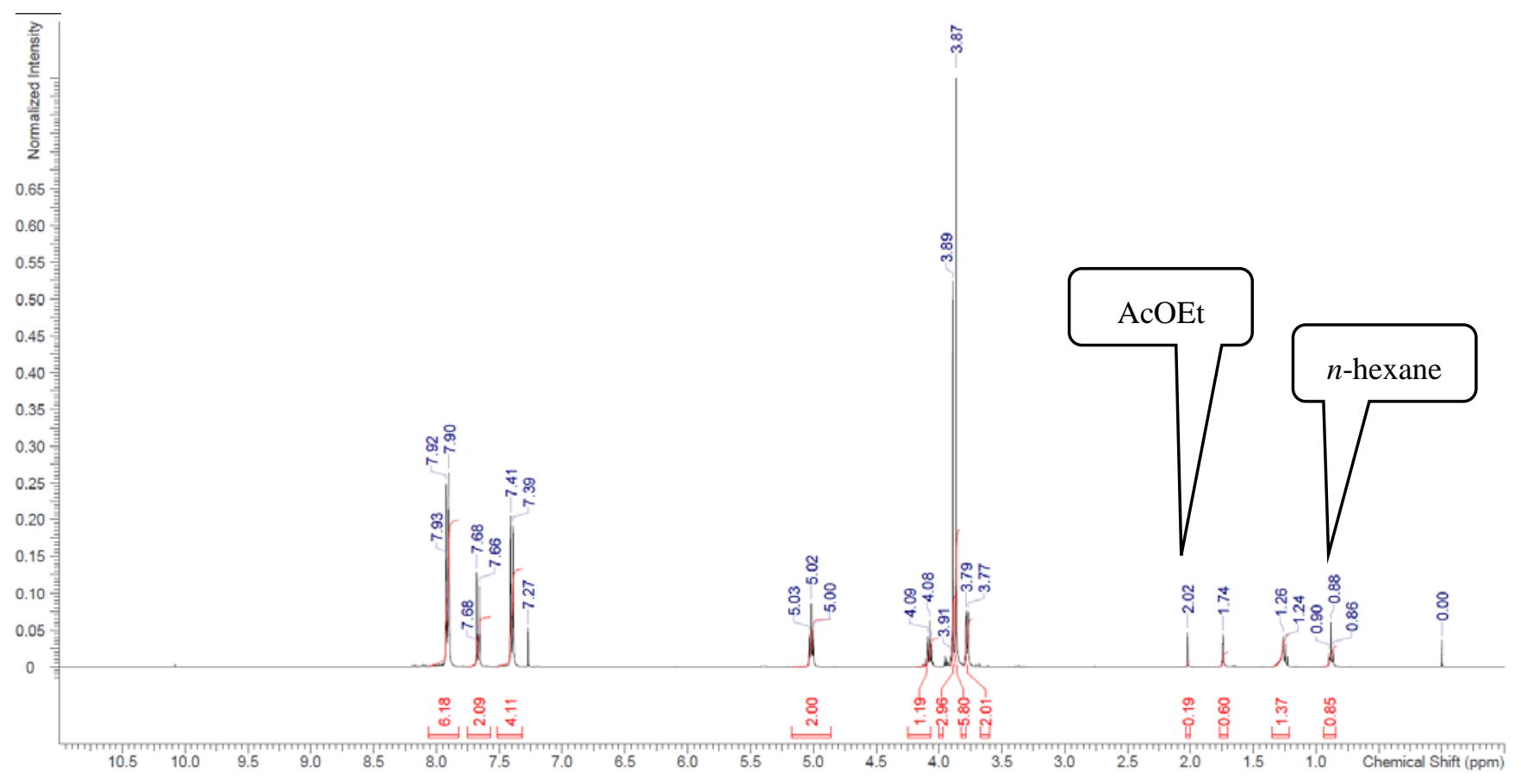




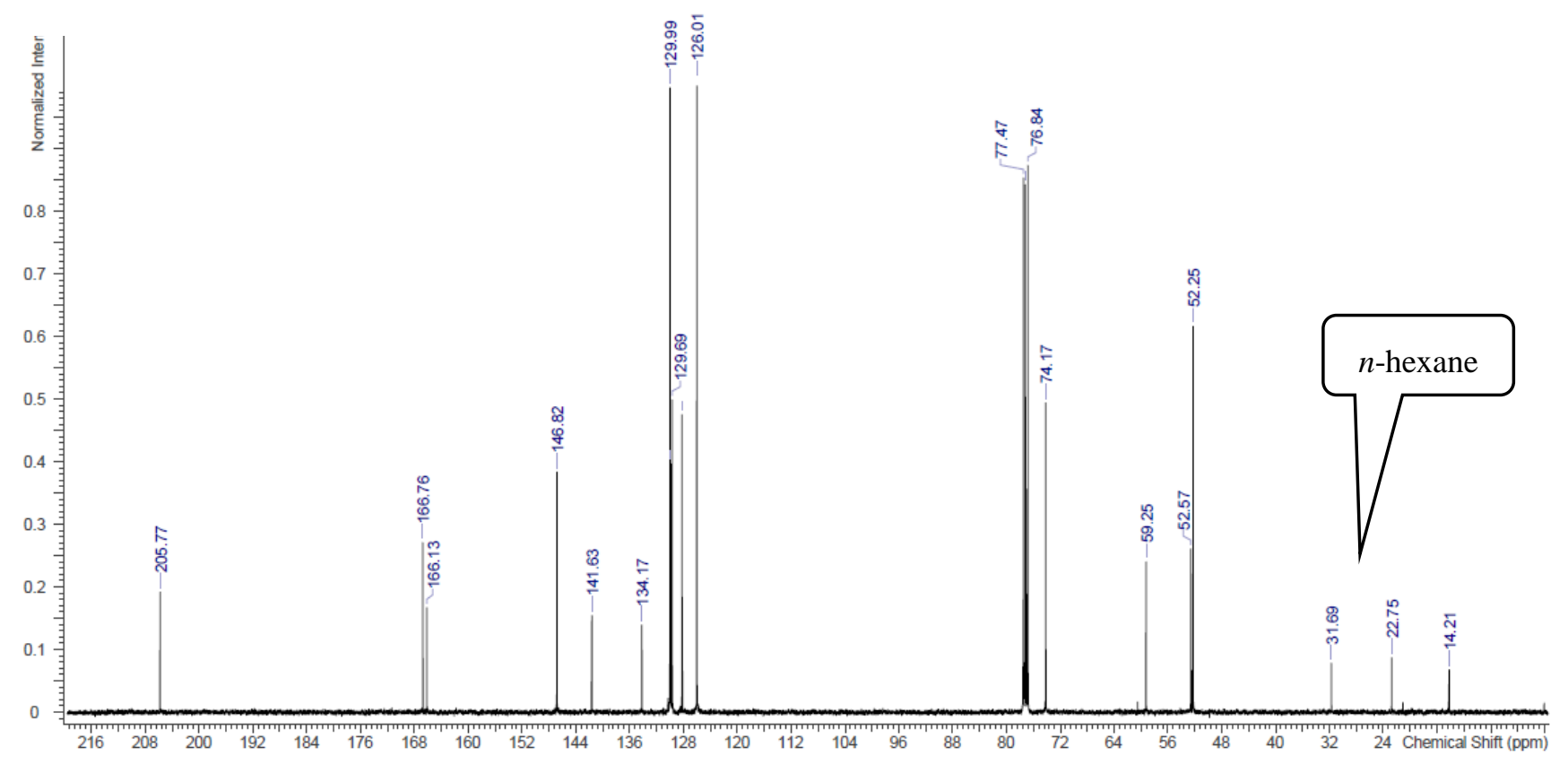




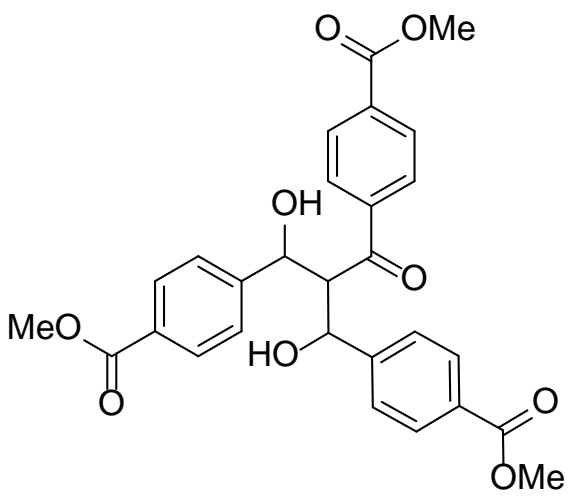

\section{Compound 3a; ${ }^{1} \mathrm{H}-{ }^{1} \mathrm{H}$ COSY Spectrum}

$3 a$

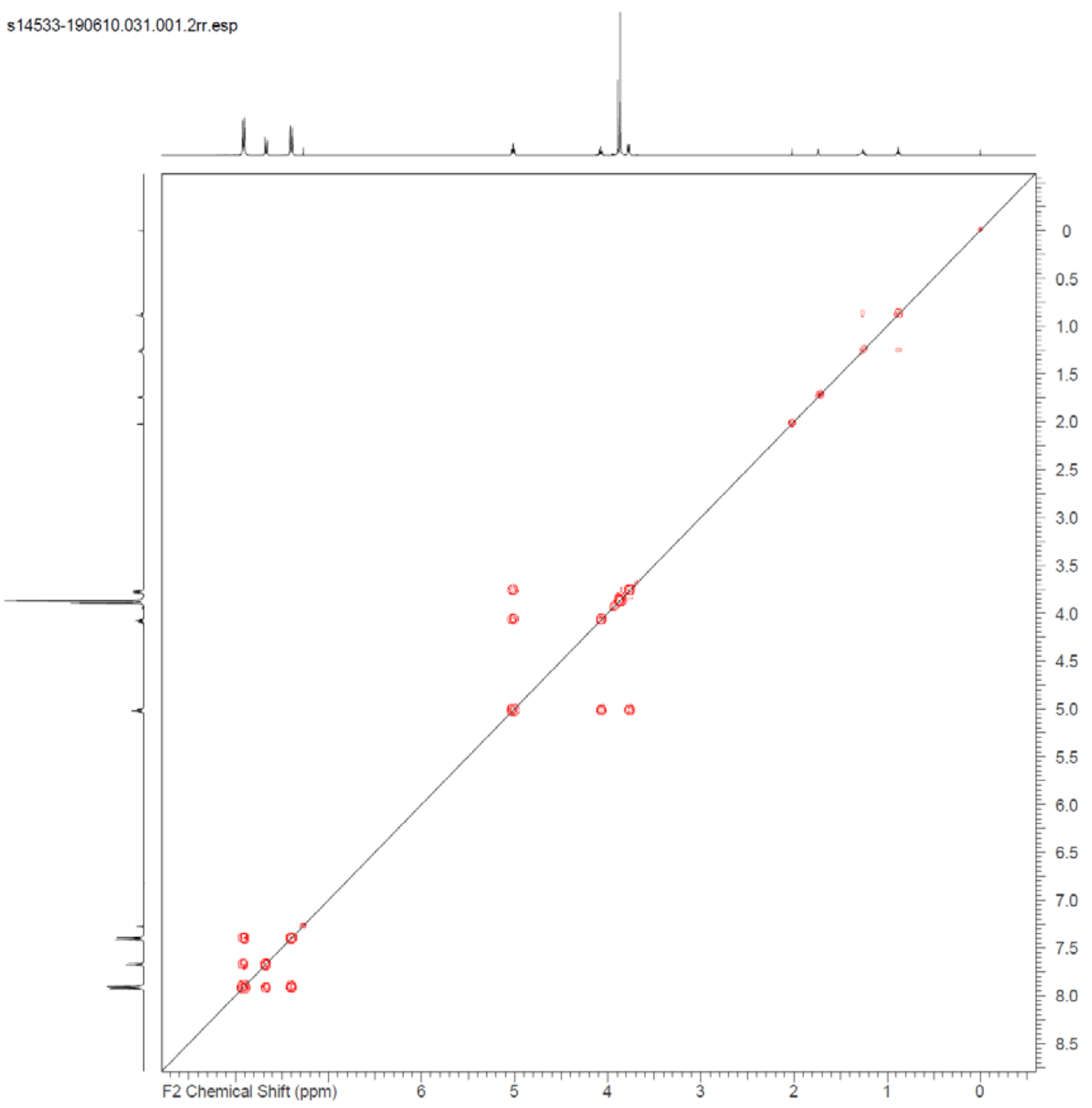




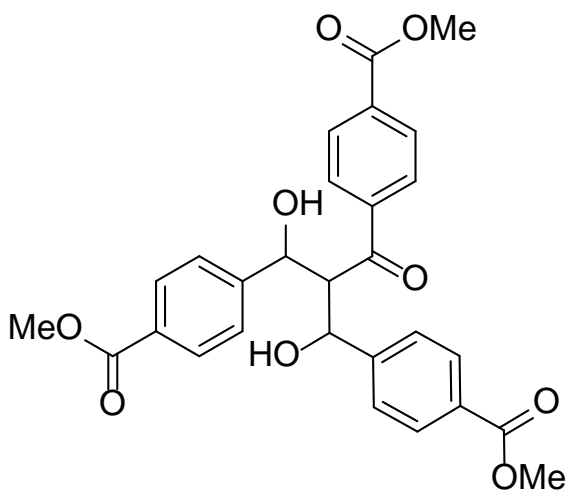

Compound 3a; ${ }^{1} \mathrm{H}-{ }^{13} \mathrm{C}$ HMBC Spectrum

$3 a$

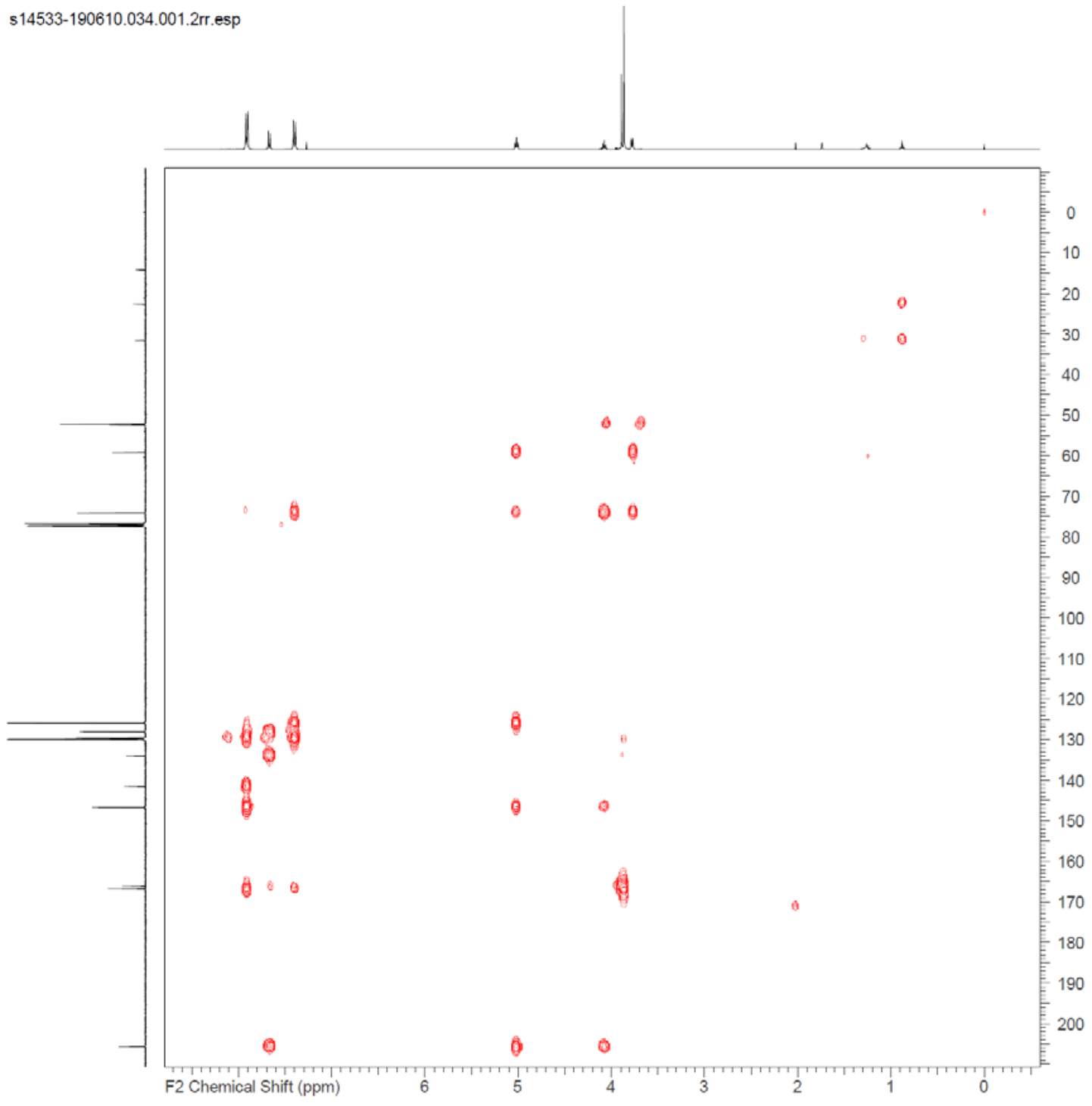




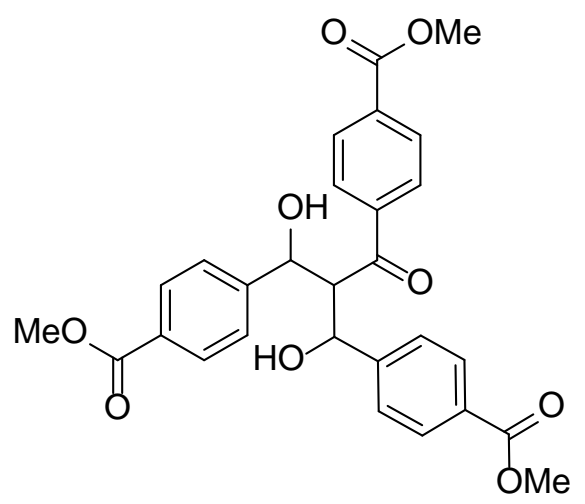

Compound 3a; ${ }^{1} \mathrm{H}-{ }^{13} \mathrm{C}$ HSQC Spectrum

$3 a$

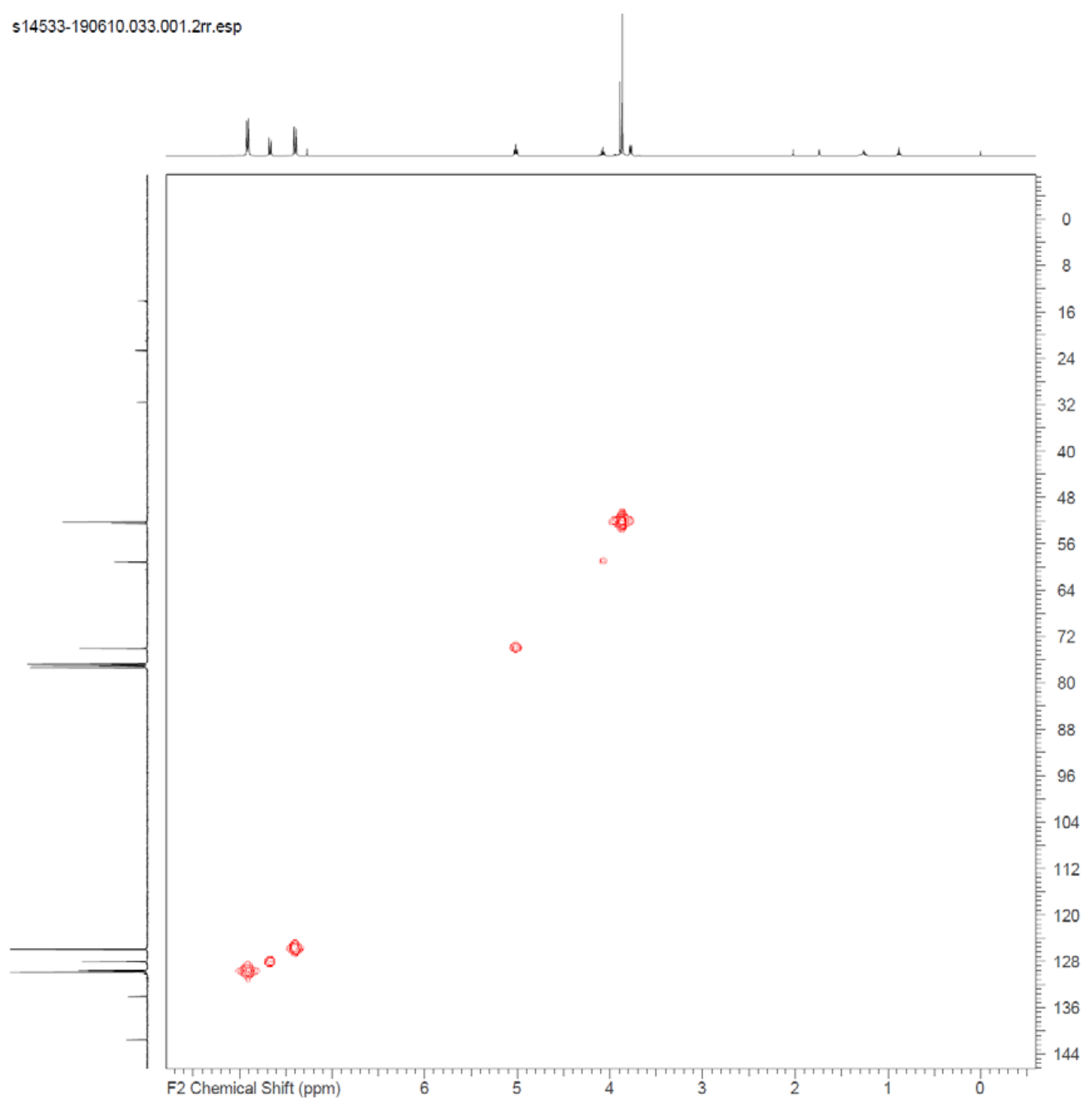




\section{Input amount and isolated yield of $2 b-2 h$ with continuous flow synthesis}

\section{1-(4-Methoxyphenyl)ethan-1-ol (2b)}

Input amount of the starting material: $829.3 \mathrm{mg}$. Isolated amount of $\mathbf{2 b}$ was $857.0 \mathrm{mg}$ (92\%)

${ }^{1} \mathrm{H}$ NMR (400 MHz, $\left.\mathrm{CDCl}_{3}\right) \delta$ 7.30-7.25 (2H, m), 6.88-6.85 (2H, m), 4.83 (1H, q, J = 6.4 Hz), 3.79 (3H, s), $1.98(1 \mathrm{H}, \mathrm{s}), 1.46(3 \mathrm{H}, \mathrm{d}, J=6.4 \mathrm{~Hz}) .{ }^{13} \mathrm{C} \mathrm{NMR}\left(100 \mathrm{MHz}, \mathrm{CDCl}_{3}\right) \delta 159.09,138.17,126.77,113.97$, 70.04, 55.39, 25.11. MS (ESI $\left.{ }^{+}\right) \mathrm{m} / \mathrm{z} 135[\mathrm{M}-\mathrm{OH}]^{+}$.

\section{4-(1-Hydroxyethyl)benzonitrile (2c)}

As for 2c, continuous flow synthesis could not be conducted due to precipitation of reaction mixture. Therefore, 2c was isolated from the reaction mixture obtained by batch synthesis.

${ }^{1} \mathrm{H}$ NMR (400 MHz, $\mathrm{CDCl}_{3}$ ) $\delta$ 7.65-7.62 (2H, m), 7.50-7.48 (2H, m), 4.96 (1H, qd, J = 6.5, 3.7 Hz), 2.07 $(1 \mathrm{H}, \mathrm{d}, J=3.7 \mathrm{~Hz}), 1.50(3 \mathrm{H}, \mathrm{d}, J=6.5 \mathrm{~Hz}) .{ }^{13} \mathrm{C} \mathrm{NMR}\left(100 \mathrm{MHz}, \mathrm{CDCl}_{3}\right) \delta 151.10,132.34,128.06$, 118.83, 111.10, 69.65, 25.39. MS $\left(\mathrm{ESI}^{+}\right) \mathrm{m} / \mathrm{z} 130[\mathrm{M}-\mathrm{OH}]^{+}$.

\section{1-(4-Nitrophenyl)ethan-1-ol (2d)}

As for $\mathbf{2 d}$, continuous flow synthesis could not be conducted due to precipitation of reaction mixture. Therefore, isolating 2d from the reaction mixture obtained by batch synthesis was tried. However, reaction mixture was complex mixture, and 2d could not be isolated. An authentic sample was purchased from a commercial source.

${ }^{1} \mathrm{H}$ NMR (400 MHz, $\left.\mathrm{CDCl}_{3}\right) \delta$ 8.20-8.17 (2H, m), 7.56-7.52 (2H, m), $5.02(1 \mathrm{H}, \mathrm{q}, J=6.5 \mathrm{~Hz}), 2.21(1 \mathrm{H}$, s), $1.52(3 \mathrm{H}, \mathrm{d}, J=6.5 \mathrm{~Hz}) .{ }^{13} \mathrm{C}$ NMR $\left(100 \mathrm{MHz} \mathrm{CDCl}_{3}\right) \delta 152.23,147.32,126.25,123.86,69.61,25.60$. $\mathrm{MS}\left(\mathrm{ESI}^{-}\right) \mathrm{m} / \mathrm{z} 166[\mathrm{M}-\mathrm{H}]^{-}$.

\section{1-(4-Bromophenyl)ethan-1-ol (2e)}

Input amount of the starting material: $1126.9 \mathrm{mg}$. Isolated amount of 2e was $1188.4 \mathrm{mg}(97 \%)$ ${ }^{1} \mathrm{H}$ NMR (400 MHz, $\left.\mathrm{CDCl}_{3}\right) \delta$ 7.47-7.43 (2H, m), 7.24-7.20 (2H, m), $4.83(1 \mathrm{H}, \mathrm{q}, J=6.5 \mathrm{~Hz}), 2.13$ (1H, s), $1.44(3 \mathrm{H}, \mathrm{d}, J=6.5 \mathrm{~Hz}) .{ }^{13} \mathrm{C} \mathrm{NMR}\left(100 \mathrm{MHz} \mathrm{CDCl}_{3}\right) \delta 144.90,131.66,127.27,121.25,69.85,25.33$. $\mathrm{MS}\left(\mathrm{ESI}^{+}\right) \mathrm{m} / \mathrm{z}$ 183, $185[\mathrm{M}-\mathrm{OH}]+$.

\section{Methyl 2-(4-(1-hydroxyethyl)phenyl)acetate (2f)}

Input amount of the starting material: $1085.3 \mathrm{mg}$. Isolated amount of $2 \mathbf{f}$ was $933.2 \mathrm{mg}$ (79\%)

${ }^{1} \mathrm{H}$ NMR (400 MHz, $\mathrm{CDCl}_{3}$ ) $\delta$ 7.33-7.31 (2H, m), 7.26-7.24 (2H, m), 4.87 (1H, q, J = 6.4 Hz), 3.68 (3H, s), 3.61 (2H, s), $1.98(1 \mathrm{H}, \mathrm{s}), 1.47$ (3H, d, $J=6.5 \mathrm{~Hz}) .{ }^{13} \mathrm{C}$ NMR $\left(100 \mathrm{MHz}, \mathrm{CDCl}_{3}\right) \delta 172.16,144.87$,

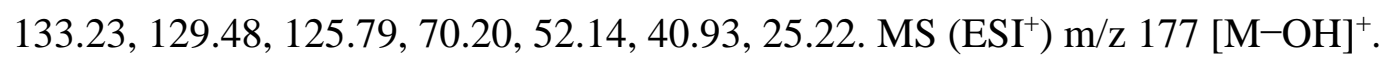




\section{Methyl 4-(1-hydroxy-2-methylpropyl)benzoate (2g)}

Input amount of the starting material: $1000.2 \mathrm{mg}$. Isolated amount of $\mathbf{2 g}$ was $645.4 \mathrm{mg}$ (51\%), which was the total amount of two samples: $120.0 \mathrm{mg}$ (97 area\%, used as an authentic sample) and $600.4 \mathrm{mg}$ (87.5 wt $\%$ by HPLC compared with the authentic sample, $600.4 \times 0.875=525.4 \mathrm{mg}$, corrected yield based on the purity).

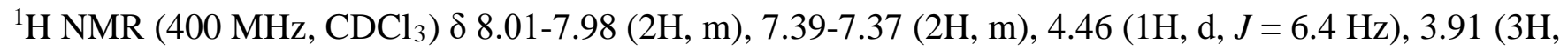
s), $2.04(1 \mathrm{H}, \mathrm{s}), 1.96$ (1H, sepd, $J=6.6,6.6 \mathrm{~Hz}), 0.96(3 \mathrm{H}, \mathrm{d}, J=6.7 \mathrm{~Hz}), 0.83(3 \mathrm{H}, \mathrm{d}, J=6.8 \mathrm{~Hz}) .{ }^{13} \mathrm{C}$ NMR (100 MHz, $\left.\mathrm{CDCl}_{3}\right) \delta 167.16,148.95,129.61,129.34,126.63,79.48,52.19,35.45,19.04,17.90$. $\mathrm{MS}\left(\mathrm{ESI}^{+}\right) \mathrm{m} / \mathrm{z} 191[\mathrm{M}-\mathrm{OH}]^{+}$.

\section{Methyl 4-(hydroxy(phenyl)methyl)benzoate (2h)}

Input amount of the starting material: $1000.2 \mathrm{mg}$. Isolated amount of $\mathbf{2 h}$ was $1457.1 \mathrm{mg}$ (99\%)

${ }^{1} \mathrm{H}$ NMR (400 MHz, $\mathrm{CDCl}_{3}$ ) $\delta$ 7.98-7.95 (2H, m), 7.44-7.42 (2H, m), 7.32-7.24 (5H, m), 5.83 (1H, s), $3.87(3 \mathrm{H}, \mathrm{s}), 2.68(1 \mathrm{H}, \mathrm{d}, J=2.1 \mathrm{~Hz}) .{ }^{13} \mathrm{C} \mathrm{NMR}\left(100 \mathrm{MHz}, \mathrm{CDCl}_{3}\right) \delta 167.07,148.90,143.42,129.88$, 129.30, 128.77, 128.02, 126.77, 126.45, 75.98, 52.18. MS $\left(\mathrm{ESI}^{+}\right) \mathrm{m} / \mathrm{z} 225[\mathrm{M}-\mathrm{OH}]^{+}$. 


\section{Compound 2b; ${ }^{1} \mathrm{H}$ NMR and ${ }^{13} \mathrm{C}$ NMR spectra}
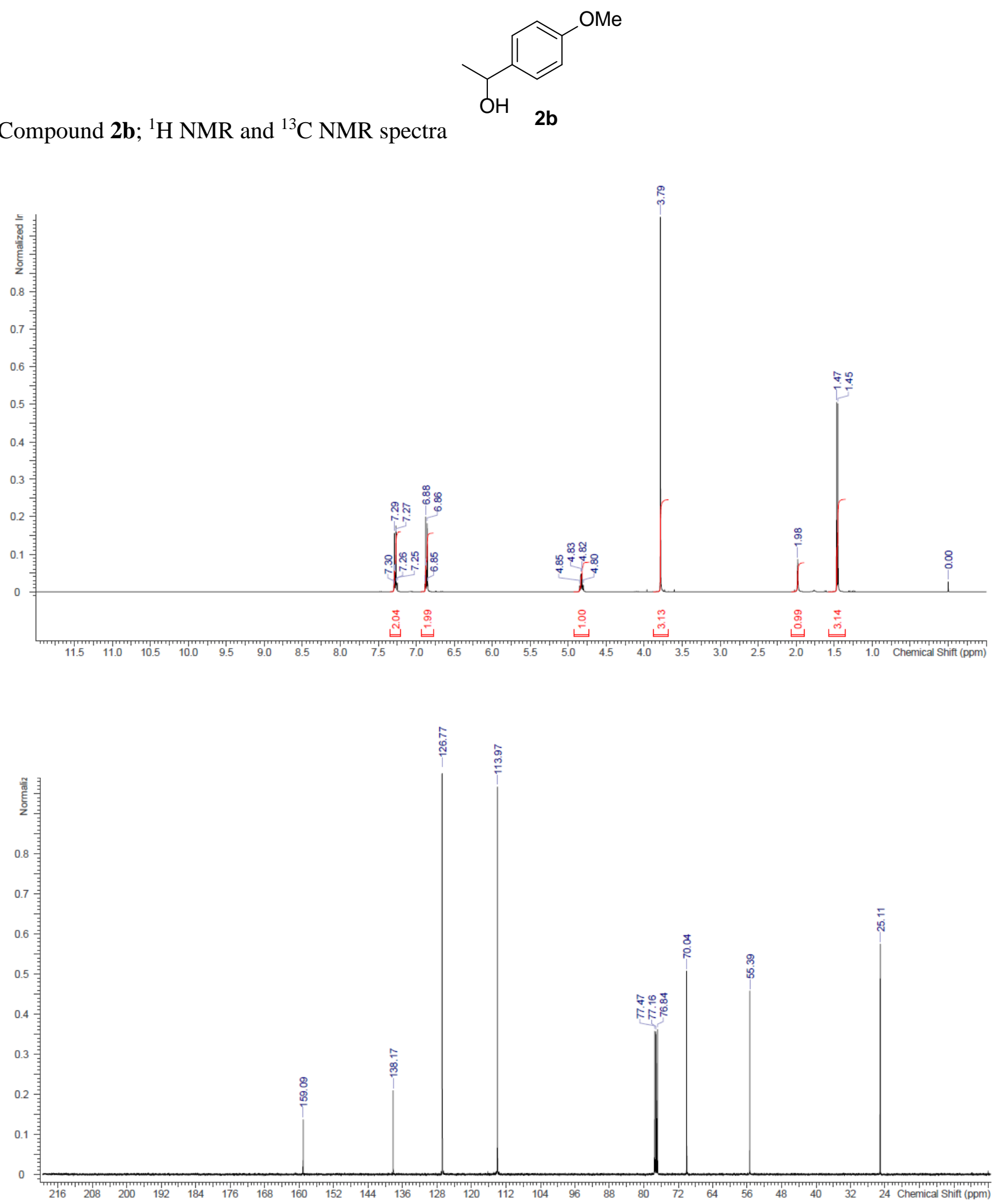
Compound 2c; ${ }^{1} \mathrm{H}$ NMR and ${ }^{13} \mathrm{C}$ NMR spectra
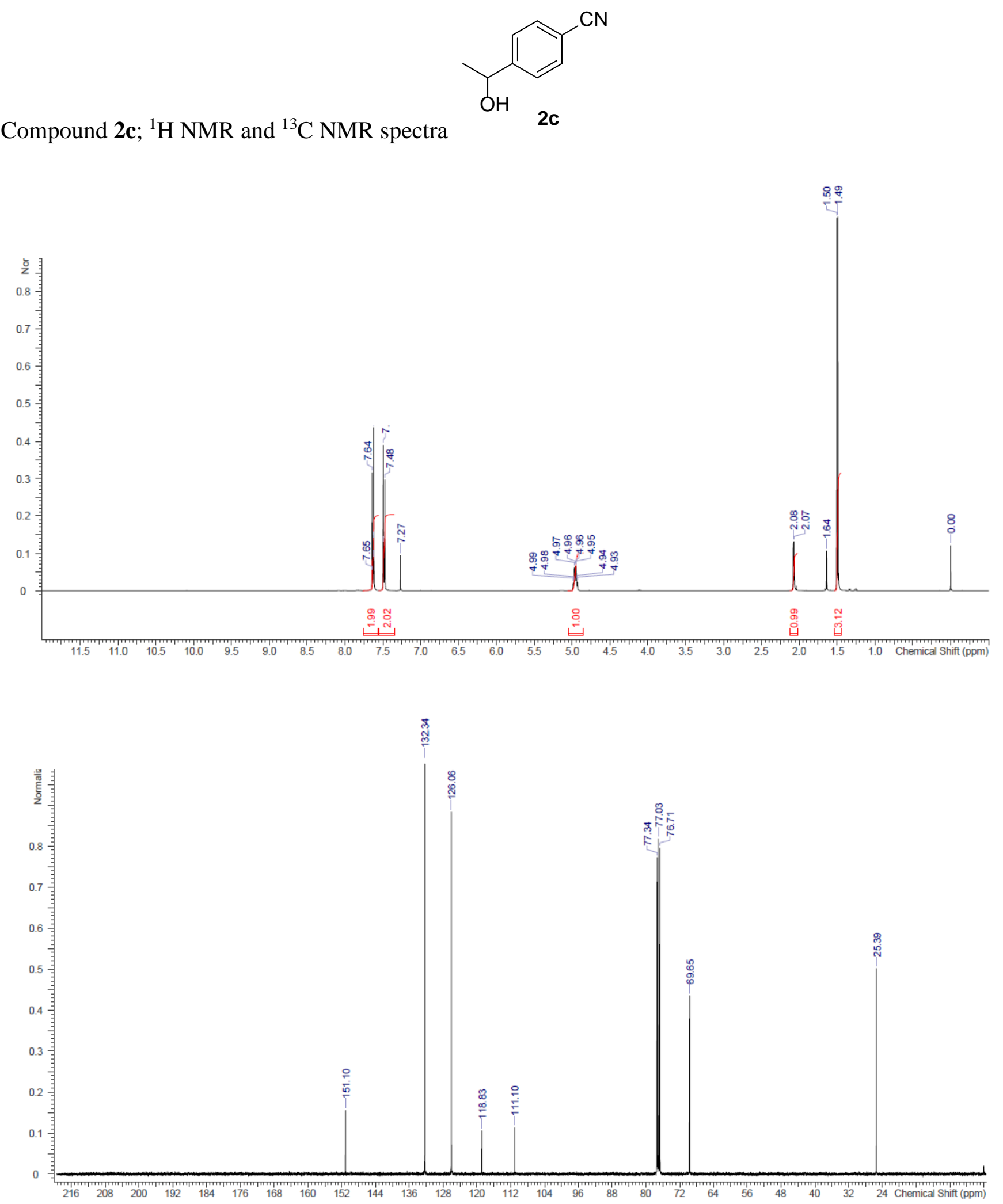


\section{Compound 2d; ${ }^{1} \mathrm{H}$ NMR and ${ }^{13} \mathrm{C}$ NMR spectra}
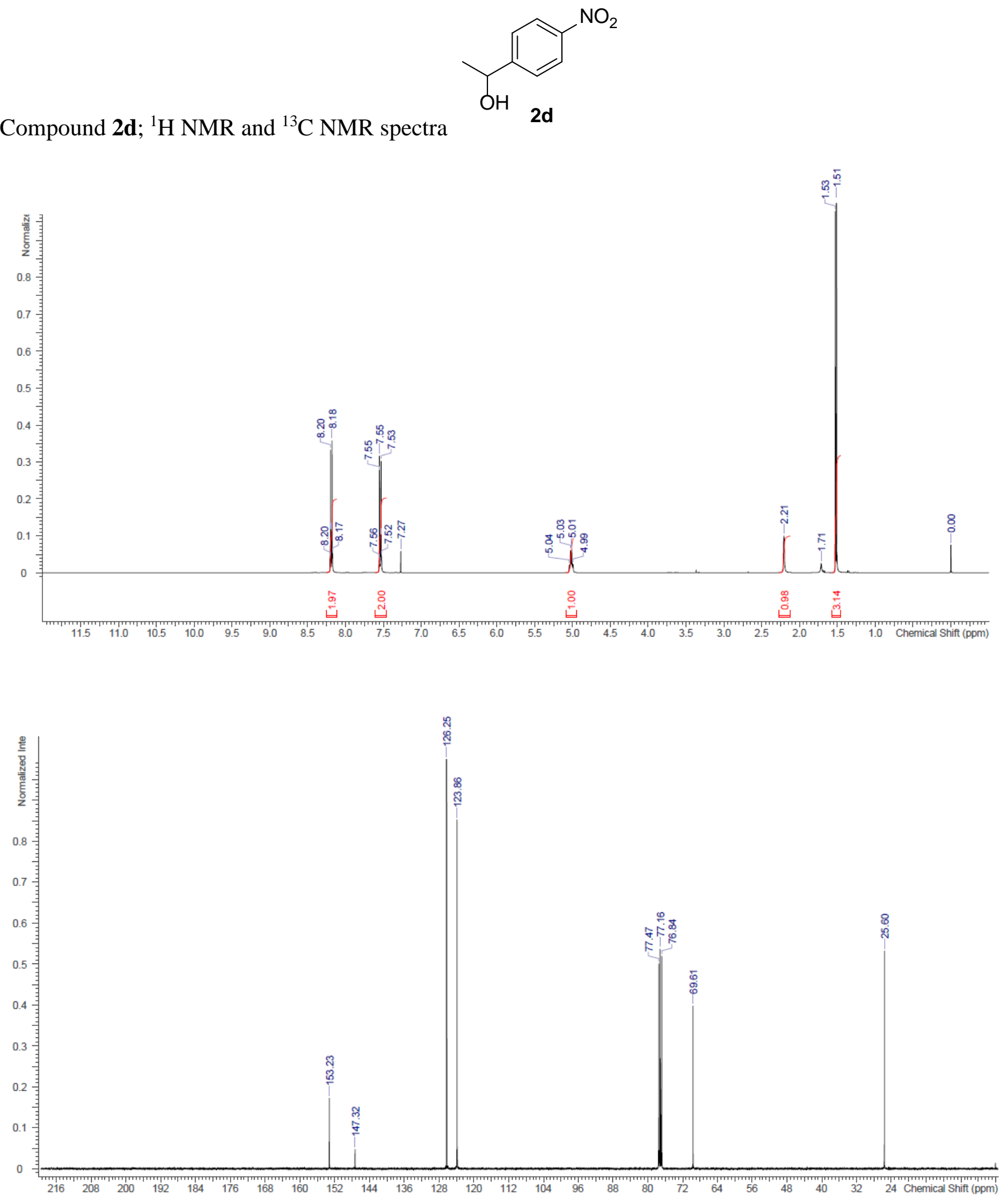
Compound 2e; ${ }^{1} \mathrm{H}$ NMR and ${ }^{13} \mathrm{C}$ NMR spectra
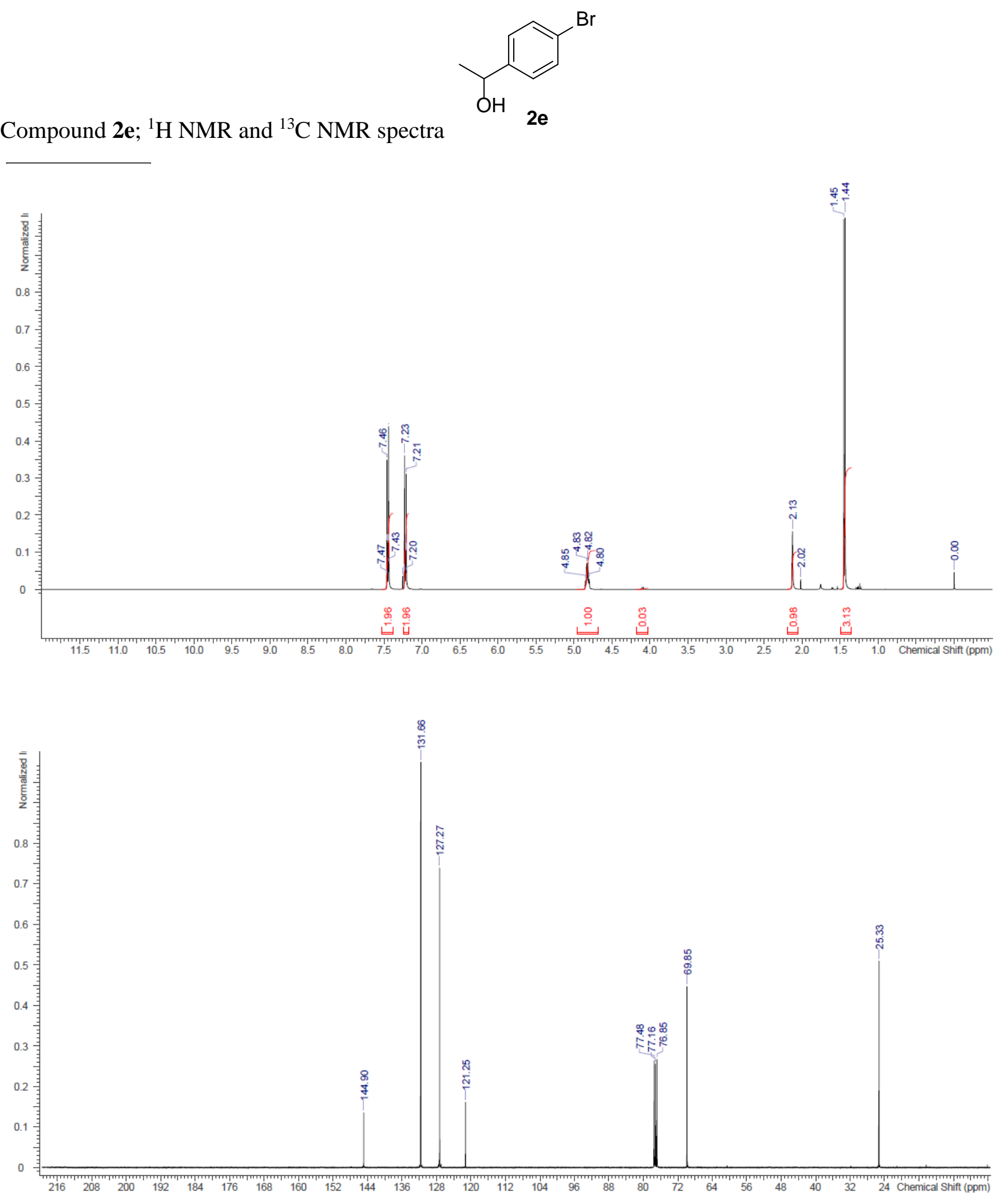


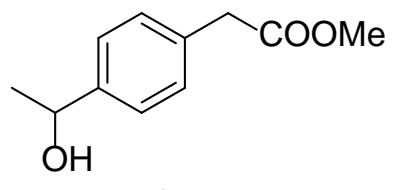

Compound 2f; ${ }^{1} \mathrm{H}$ NMR and ${ }^{13} \mathrm{C}$ NMR spectra

$2 f$
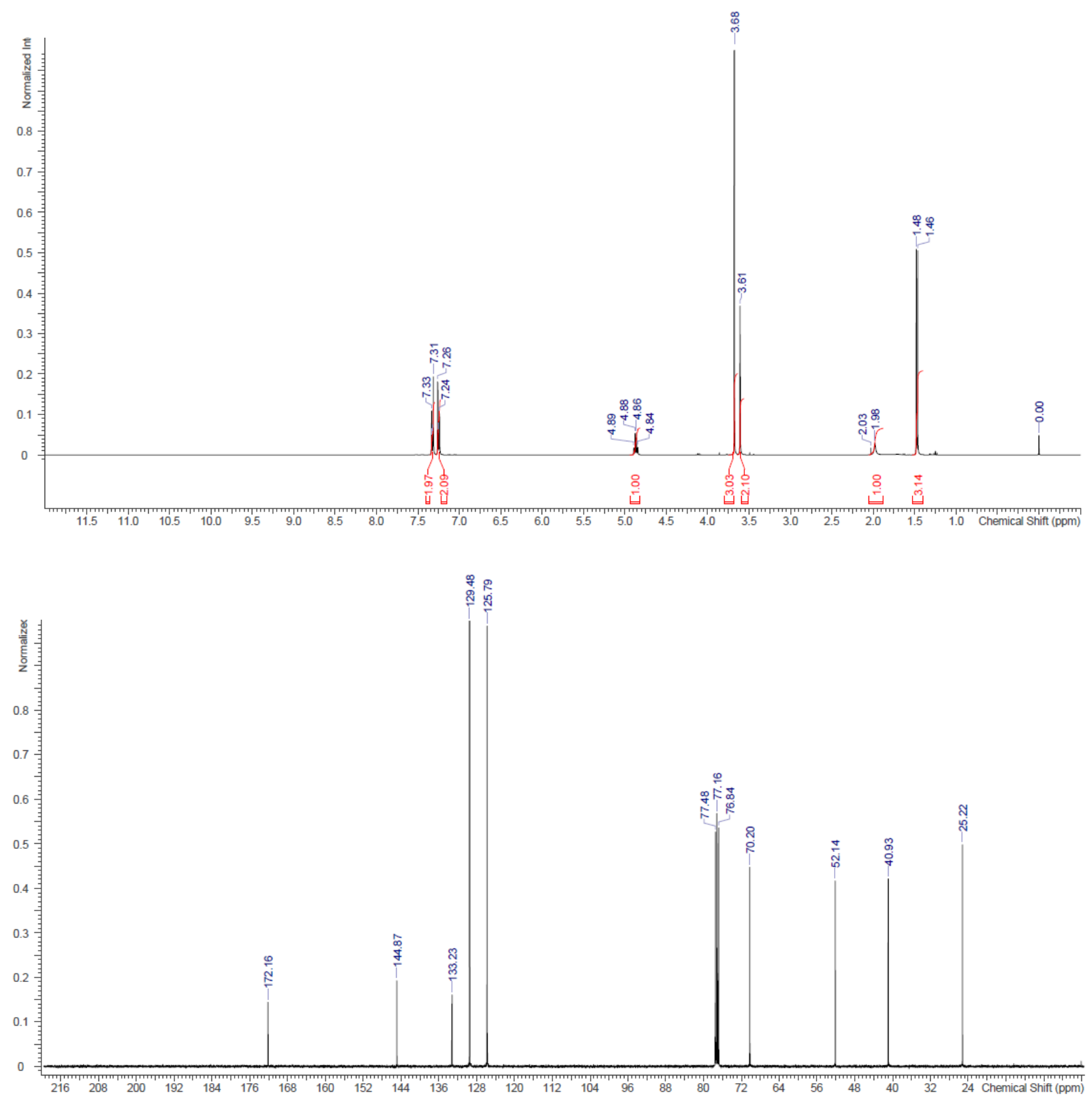
Compound 2g; ${ }^{1} \mathrm{H}$ NMR and ${ }^{13} \mathrm{C}$ NMR spectra
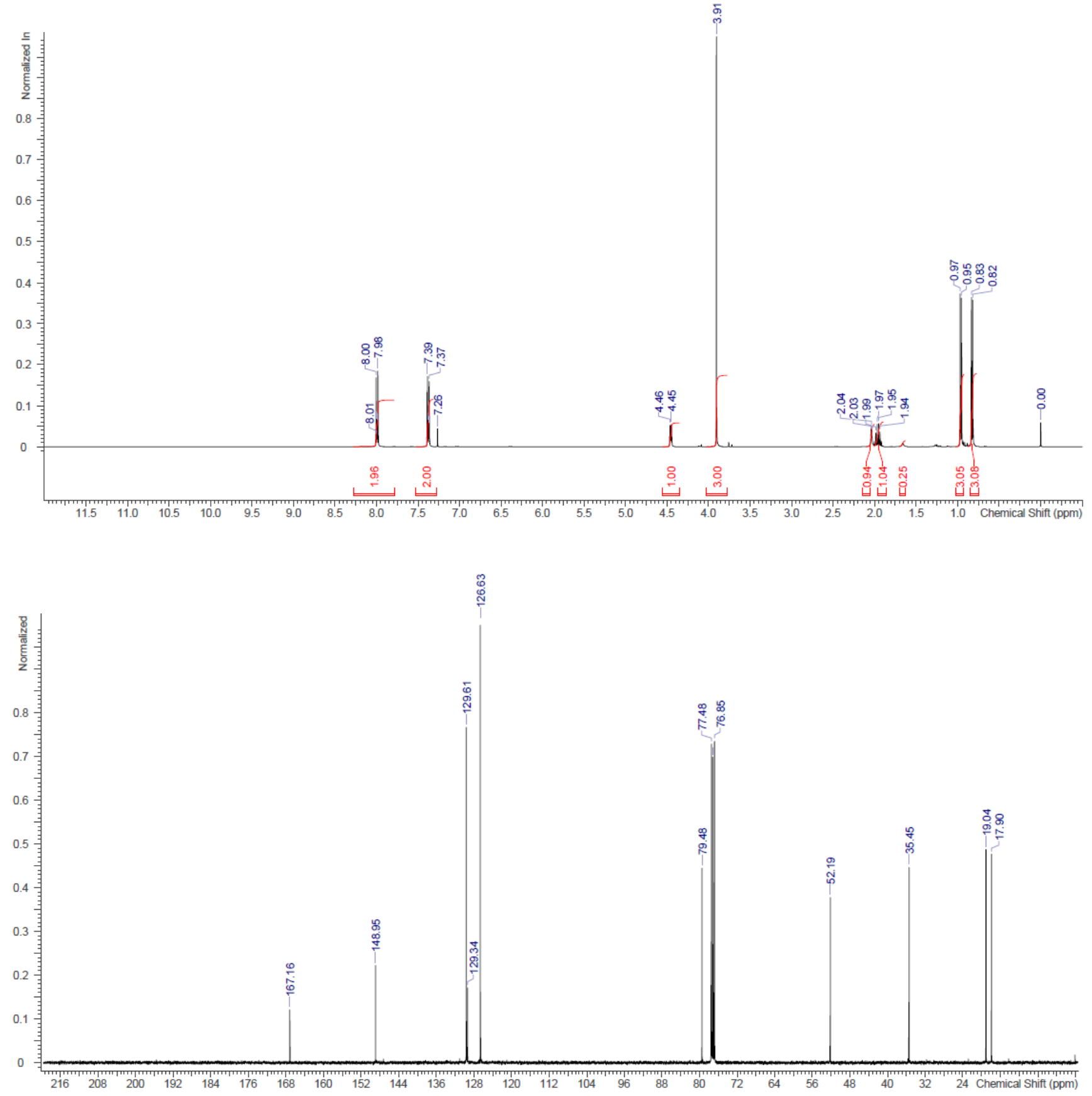
Compound $2 \mathbf{h} ;{ }^{1} \mathrm{H}$ NMR and ${ }^{13} \mathrm{C}$ NMR spectra
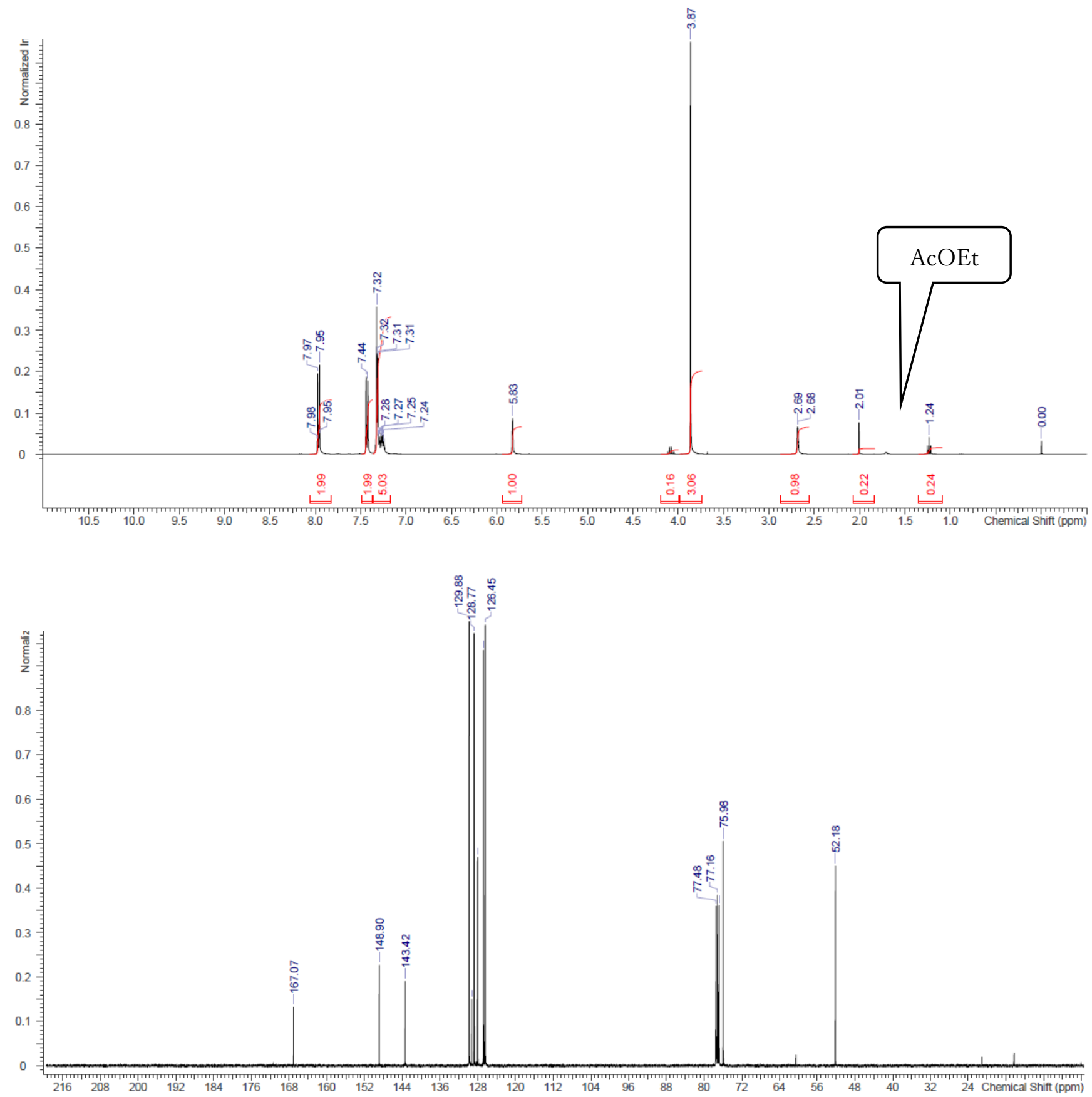


\section{Heat flow about addition of MeMgBr to 1a}

Table S1. Calorimetry analysis result about addition of MeMgBr to 1a

\begin{tabular}{|c|c|c|c|c|c|c|c|c|c|c|}
\hline \multirow[t]{2}{*}{ Addition of $\mathrm{MeMgBr}$} & \multirow{2}{*}{$\begin{array}{c}\text { Starting } \\
\text { Material } \\
\text { (g) }\end{array}$} & \multirow{2}{*}{$\begin{array}{c}\text { Reaction } \\
\text { Heat } \\
\text { Conversion } \\
(\%)\end{array}$} & \multirow{2}{*}{\begin{tabular}{|c|} 
Enthalpy \\
$(\mathrm{kJ})$
\end{tabular}} & \multirow{2}{*}{$\begin{array}{c}\text { Reaction } \\
\text { Mass } \\
\text { (kg) }\end{array}$} & \multirow{2}{*}{$\begin{array}{l}\text { Process } \\
\text { Temp } \\
\text { (degC) }\end{array}$} & \multirow{2}{*}{$\begin{array}{c}\Delta \mathrm{Tad} \\
\text { (K) }\end{array}$} & \multirow{2}{*}{$\begin{array}{c}\mathrm{cpr} \\
(\mathrm{kJ} /(\mathrm{kg} \cdot \mathrm{K}))\end{array}$} & \multicolumn{2}{|c|}{ Reaction Heat } & \multirow[t]{2}{*}{ Methods } \\
\hline & & & & & & & & $(\mathrm{kJ} / \mathrm{mol})$ & $(\mathrm{kJ} / \mathrm{kg})$ & \\
\hline- & 5.0 & 100 & 5.6 & 0.048 & 25 & 68 & $1.70 \rightarrow 1.70$ & 184 & 115 & $\begin{array}{c}\text { RTCal } \\
\text { (rtc) }\end{array}$ \\
\hline
\end{tabular}

Molecular weight of the starting material 164.16
Reaction Mass: Input Mass of a solution of the starting material cpr: Specific heat of THF

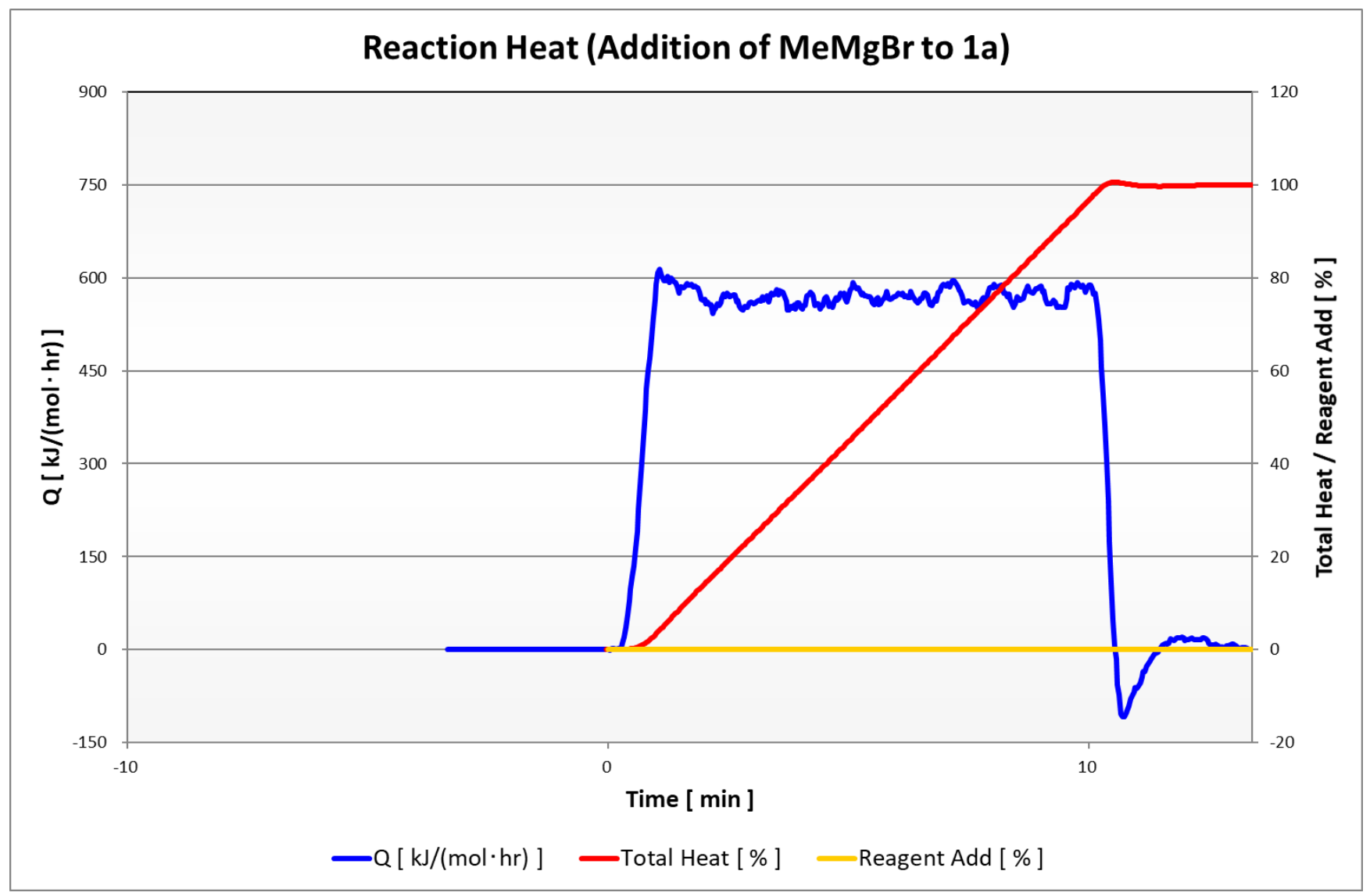

Figure S1. Heat flow chart about addition of MeMgBr to 1a 


\section{Justification of calorimetry analysis combined with continuous flow system and RC1e}

Justification of calorimetry analysis combined with continuous flow system and RC1e was demonstrated. Neutralization reaction with $\mathrm{NaOH}$ and $\mathrm{HCl}$ was actually measured. The experimental procedure was described as follows.

Two solutions were fed by the Vapourtec V-3; solution A was an aqueous solution of $\mathrm{NaOH}(1.0$ $\mathrm{mmol} / \mathrm{mL})$ and solution B was an aqueous solution of $\mathrm{HCl}(1.0 \mathrm{mmol} / \mathrm{mL})$. Flow rate of solution A was set as $1.00 \mathrm{~mL} / \mathrm{min}$, and that of solution $\mathrm{B}$ was set as $1.00 \mathrm{~mL} / \mathrm{min}$ so that the equivalent of $\mathrm{HCl}$ would be adjusted to 1.0 equiv. Two solutions were mixed at a T-shape mixer, and the combined solution was passed through PFA tube reactors (inner diameter $1.0 \mathrm{~mm}$, internal volume $7.85 \mathrm{~mL}$ ). The flow system consisting of T-mixer and tube reactor was put in RC1e and RC1e was filled with water (475 mL). Filled water was adjusted to $25^{\circ} \mathrm{C}$, and heat generation was measured for 30 minutes. Input amount of the starting material $\mathrm{NaOH}$ was defined as $1.20 \mathrm{~g}(1.0 \mathrm{mmol} / \mathrm{mL} \times 1.00 \mathrm{~mL} / \mathrm{min} \times 30 \mathrm{~min} \times 40 \mathrm{mg} / \mathrm{mmol})$

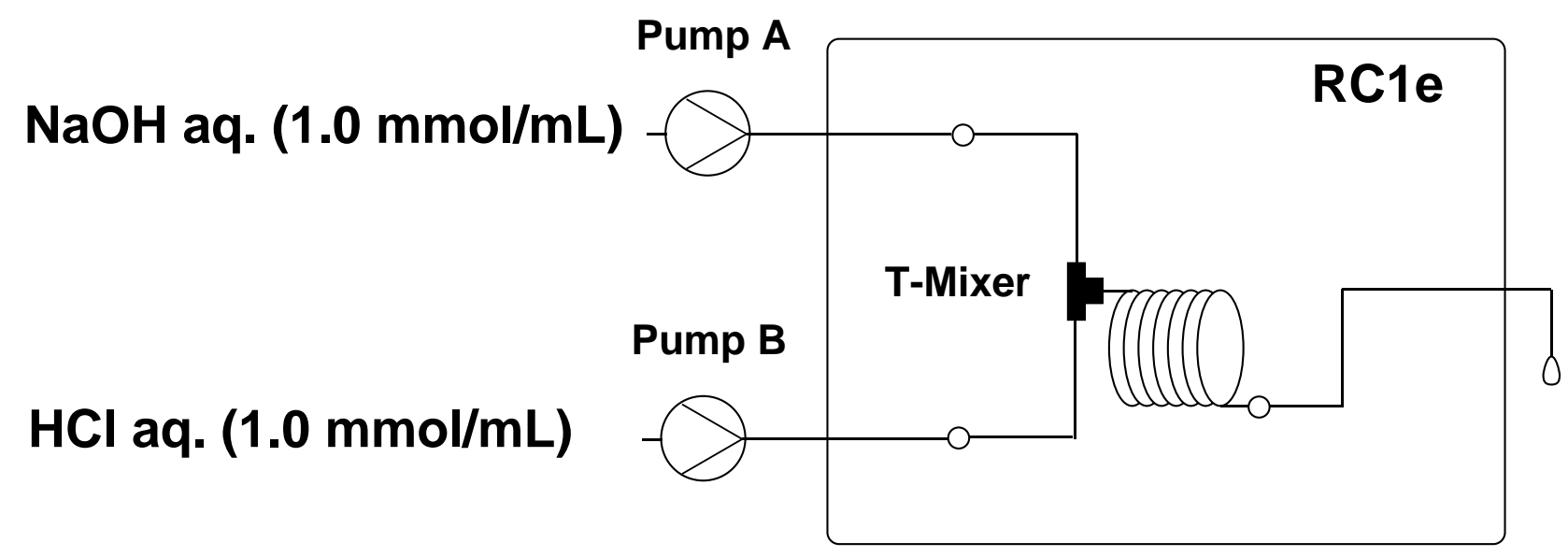

Figure S2. Calorimetry analysis instrument combined with continuous flow system and RC1e

Table S2. Calorimetry analysis result about neutralization reaction with $\mathrm{NaOH}$ and $\mathrm{HCl}$

\begin{tabular}{|c|c|c|c|c|c|c|c|c|c|c|}
\hline \multirow{2}{*}{ Flow HClaq.+NaOHaq. } & \multirow{2}{*}{$\begin{array}{c}\text { Starting } \\
\text { material } \\
(\mathrm{NaOH}) \\
(\mathrm{g})\end{array}$} & \multirow{2}{*}{$\begin{array}{c}\text { Reaction } \\
\text { Heat } \\
\text { Conversion } \\
(\%)\end{array}$} & \multirow{2}{*}{$\begin{array}{c}\text { Enthalpy } \\
\text { (kJ) }\end{array}$} & \multirow{2}{*}{$\begin{array}{c}\text { Reaction } \\
\text { Mass } \\
(\mathrm{kg})\end{array}$} & \multirow{2}{*}{$\begin{array}{c}\text { Process } \\
\text { Temp } \\
\text { (degC) }\end{array}$} & \multirow{2}{*}{$\begin{array}{c}\Delta \mathrm{Tad} \\
(\mathrm{K}) \\
\end{array}$} & \multirow{2}{*}{$\begin{array}{c}\mathrm{cpr} \\
(\mathrm{kJ} /(\mathrm{kg} \cdot \mathrm{K}))\end{array}$} & \multicolumn{2}{|c|}{ Reaction Heat } & \multirow{2}{*}{ Methods } \\
\hline & & & & & & & & $(\mathrm{kJ} / \mathrm{mol})$ & $(\mathrm{kJ} / \mathrm{kg})$ & \\
\hline- & 1.2 & 100 & 1.73 & 0.030 & 25 & 12.6 & $4.58 \rightarrow 4.58$ & 58 & 58 & $\begin{array}{l}\text { RTCal } \\
(r \mathrm{tc})\end{array}$ \\
\hline
\end{tabular}




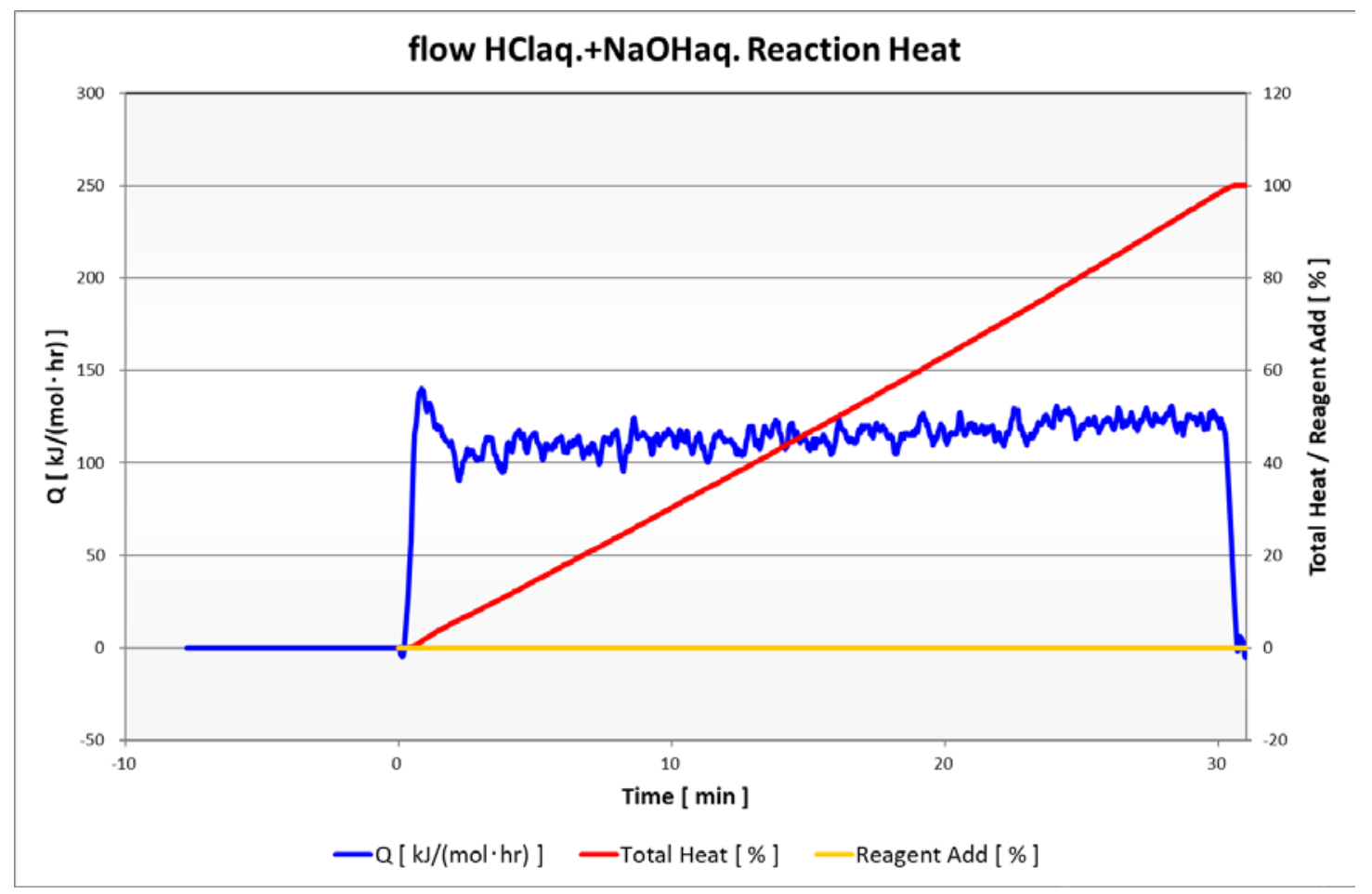

Figure S3. Heat flow chart about neutralization reaction with $\mathrm{NaOH}$ and $\mathrm{HCl}$

Measured value was $58 \mathrm{~kJ} / \mathrm{mol}$, which was almost equivalent to the reported value $(56.5 \mathrm{~kJ} / \mathrm{mol}$ from The Chemical Society of Japan (ed.), Kagaku Binran, Kisohen, part II, 5th edition, Maruzen, Tokyo, 2004, p.289, in Japanese). This result indicated that this calorimetry analysis system was justified. 
Simulation details based on the heat of reaction

Table S3. Estimation of addition time of MeMgBr by simulation of internal temperature

\begin{tabular}{|l|l|l|l|l|}
\hline entry & $\begin{array}{l}\text { charge amount of } \\
\mathbf{1 a}\end{array}$ & $\begin{array}{l}\text { internal } \\
\text { temperature } \\
\left({ }^{\circ} \mathrm{C}\right)\end{array}$ & $\begin{array}{l}\text { jacket } \\
\text { temperature } \\
\left({ }^{\circ} \mathrm{C}\right)\end{array}$ & $\begin{array}{l}\text { required addition } \\
\text { time } \\
(\mathrm{min})\end{array}$ \\
\hline 1 & $5.0 \mathrm{~g}$ & 25 & -20 & 2 \\
\hline 2 & $10 \mathrm{~kg}$ & 25 & -20 & 30 \\
\hline 3 & $50 \mathrm{~kg}$ & 25 & -20 & 45 \\
\hline 4 & $300 \mathrm{~kg}$ & 25 & -20 & 90 \\
\hline
\end{tabular}

$\mathrm{q}=\mathrm{U} \times \mathrm{A} \times(\mathrm{Tj}-\mathrm{Tr})$

$\mathrm{dTr} / \mathrm{d} \theta=\mathrm{q} /(\mathrm{Cp} \times \mathrm{W})$

q: Amount of heat transfer $[\mathrm{J} / \mathrm{s}]$

$\mathrm{U}$ : Overall heat transfer coefficient $\left[\mathrm{J} / \mathrm{m}^{2} \cdot \mathrm{K} \cdot \mathrm{s}\right]$

A: Heat transfer area $\left[\mathrm{m}^{2}\right]$

$\mathrm{Tj}$ : Jacket temperature [K]

Tr: Internal temperature [K]

Cp: Specific heat $[\mathrm{J} / \mathrm{kg} \cdot \mathrm{K}]$

W: Weight [kg]

$\theta:$ Time $[\mathrm{s}]$

Assumption:

The total heat of reaction: 184 [kJ/mol]

Overall heat transfer coefficient: $150 \mathrm{~J} / \mathrm{m}^{2} \cdot \mathrm{K} \cdot \mathrm{s}$ (measured with our manufacturing equipment).

The internal temperature is maintained at $25 \pm 5^{\circ} \mathrm{C}$. 
Initial conditions are shown as follows.

Table S4. Initial conditions for estimation of addition time of $\mathrm{MeMgBr}$

\begin{tabular}{|l|l|l|l|l|}
\hline- & entry 1 & entry 2 & entry 3 & entry 4 \\
\hline $\begin{array}{l}\text { Diameter of batch } \\
\text { reactor [m] }\end{array}$ & 0.05 & 0.6 & 1.2 & 1.8 \\
\hline $\begin{array}{l}\text { Initial volume } \\
{\left[\mathrm{m}^{3}\right]}\end{array}$ & 0.00005 & 0.1 & 0.5 & 3.0 \\
\hline $\begin{array}{l}\text { Internal } \\
\text { temperature }\left[{ }^{\circ} \mathrm{C}\right]\end{array}$ & 25 & 25 & 25 & 25 \\
\hline $\begin{array}{l}\text { Jacket } \\
\text { temperature }\left[{ }^{\circ} \mathrm{C}\right]\end{array}$ & -20 & -20 & -20 & -20 \\
\hline $\begin{array}{l}\text { Specific heat } \\
{[\mathrm{kJ} / \mathrm{kg} \cdot \mathrm{K}]}\end{array}$ & 1.7 & 1.7 & 1.7 & 1.7 \\
\hline Density $\left[\mathrm{kg} / \mathrm{m}^{3}\right]$ & 889.2 & 889.2 & 889.2 & 889.2 \\
\hline $\begin{array}{l}\text { Amount of } \\
\text { MeMgBr }\left[\mathrm{m}^{3}\right]\end{array}$ & 0.000029 & 0.058 & 0.29 & 1.74 \\
\hline
\end{tabular}

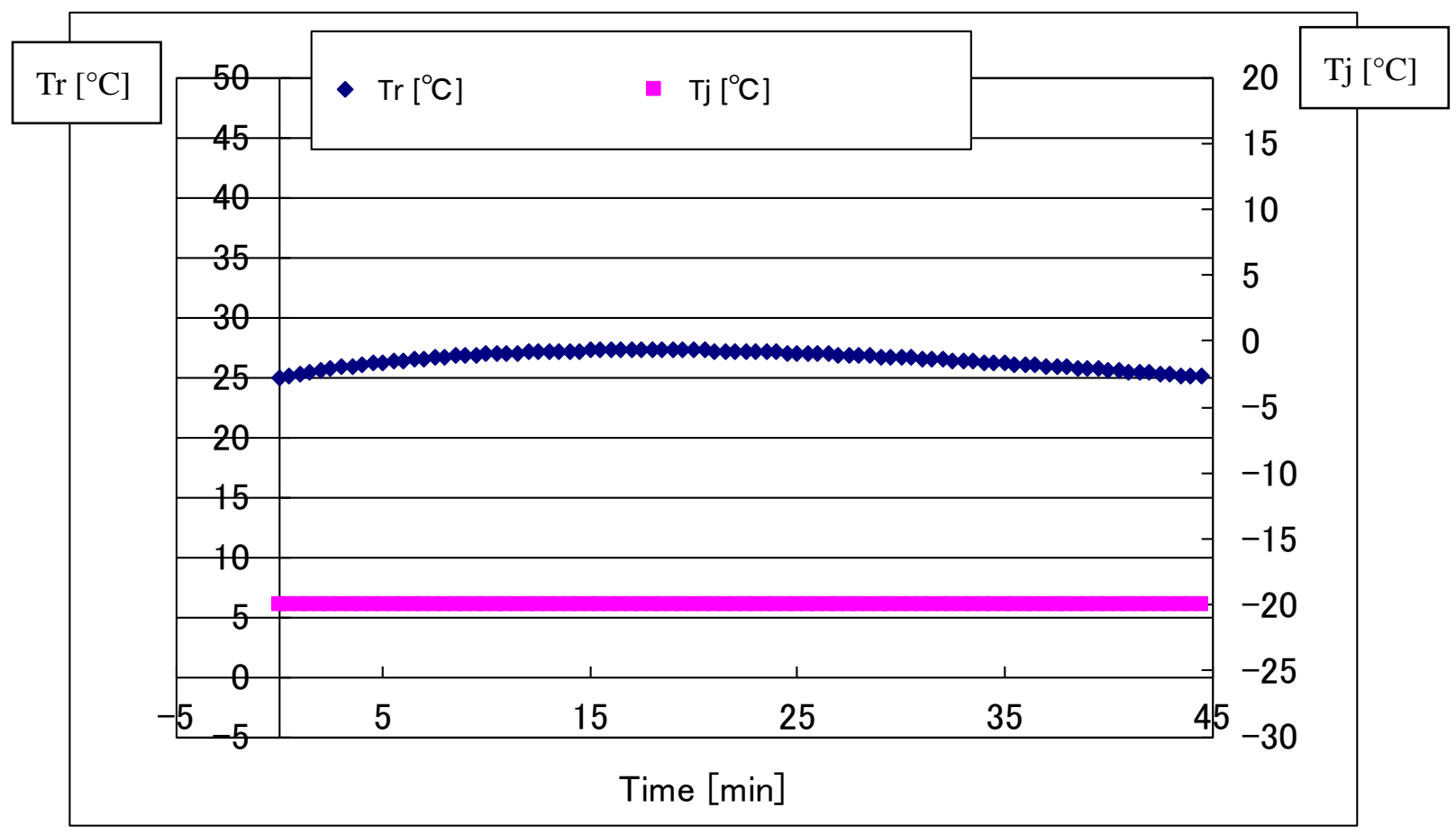

Figure S4. Representative chart of the simulation (entry 3) 
Detailed description of the equipment used for continuous flow synthesis

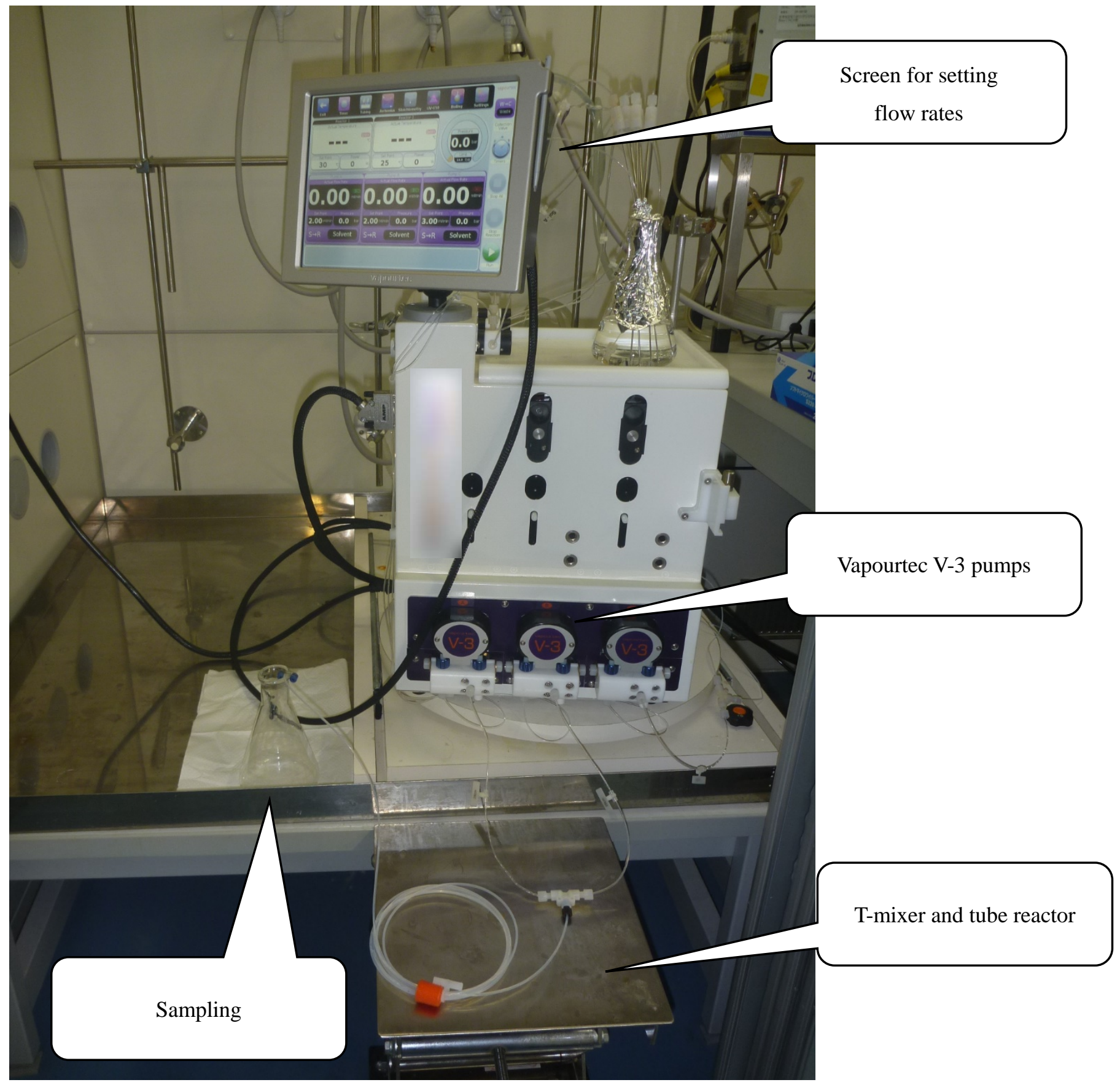

Figure S5. Detailed description of the equipment used for continuous flow synthesis -1 


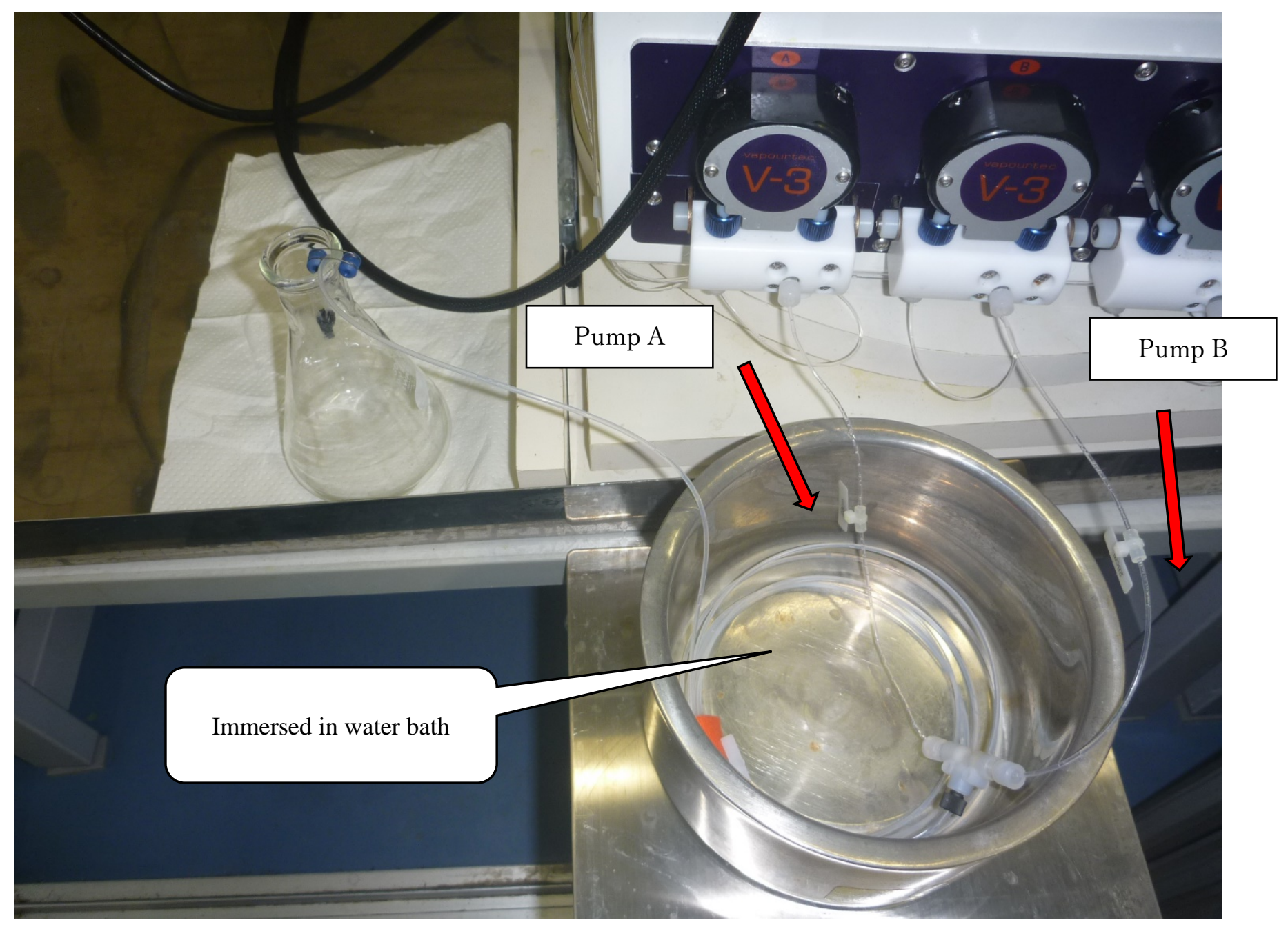

Figure S6. Detailed description of the equipment used for continuous flow synthesis -2 


\section{Procedure of running for a long duration}

Two solutions were fed by the Vapourtec V-3; solution A was prepared form 1a (120.00 g) and 10 volumes of THF (1200 mL, $0.559 \mathrm{mmol} / \mathrm{mL})$, and solution B was MeMgBr in THF $(1.02 \mathrm{mmol} / \mathrm{mL}$ and 1.06 $\mathrm{mmol} / \mathrm{mL}$ ). Flow rate of solution A was set as $3.27 \mathrm{~mL} / \mathrm{min}$, and that of solution B was set as $1.97 \mathrm{~mL} / \mathrm{min}$ in the case of $1.02 \mathrm{mmol} / \mathrm{mL}$, and $1.90 \mathrm{~mL} / \mathrm{min}$ in the case of $1.06 \mathrm{mmol} / \mathrm{mL}$ so that the equivalent of $\mathrm{MeMgBr}$ would be adjusted to 1.10 equiv. Two solutions were mixed at a T-shape mixer in a bath of $25^{\circ} \mathrm{C}$, and the combined solution was passed through PFA tube reactors (inner diameter $1.0 \mathrm{~mm}$, internal volume $2.14 \mathrm{~mL}$ ). After reaching a steady state (running for 3 minutes), running was conducted for 6 hours. In running for 6 hours, the reaction mixture was sampled according to the timetable as shown in Table 6. The sampled reaction mixture was poured into $\mathrm{MeCN} / \mathrm{H}_{2} \mathrm{O}(80 / 20(\mathrm{v} / \mathrm{v}))$ containing $0.1 \mathrm{vol} \%$ of $\mathrm{HCOOH}$ directly and diluted in a measuring flask to measure the molecular ratio (1a, 2a, 3×3a, 4a, 5a and other impurities). Pressure indicators attached to pumps showed 0.0-0.1 bar in running. 